\title{
Discontinuous Galerkin time stepping method for solving linear space fractional partial differential equations
}

\author{
Yanmei Liu ${ }^{\mathrm{a}}$, Yubin Yan*, ${ }^{*}$ Monzorul Khan ${ }^{\mathrm{c}}$ \\ ${ }^{a}$ Department of Mathematics, LuLiang University, Lishi, 033000, P.R. China \\ ${ }^{b}$ Department of Mathematics, University of Chester, $\mathrm{CH} 14 \mathrm{BJ}$ \\ ${ }^{c}$ Department of Mathematics, University of Chester, CH1 $4 \mathrm{BJ}$
}

\begin{abstract}
In this paper, we consider the discontinuous Galerkin time stepping method for solving the linear space fractional partial differential equations. The space fractional derivatives are defined by using Riesz fractional derivative. The space variable is discretized by means of a Galerkin finite element method and the time variable is discretized by the discontinuous Galerkin method. The approximate solution will be sought as a piecewise polynomial function in $t$ of degree at most $q-1, q \geq 1$, which is not necessarily continuous at the nodes of the defining partition. The error estimates in the fully discrete case are obtained and the numerical examples are given.
\end{abstract}

Key words:

Space fractional partial differential equations, discontinuous Galerkin method, finite element method, error estimates

AMS Subject Classification: 65M12; 65M06; 65M70

\section{Introduction}

In this paper we will consider the discontinuous Galerkin time stepping methods for solving the following linear space fractional partial differential equation, with $1 / 2<\alpha \leq 1$,

$$
\begin{aligned}
& \frac{\partial u(t, x)}{\partial t}-\frac{\partial^{2 \alpha} u(t, x)}{\partial|x|^{2 \alpha}}=f(t, x), \quad 0<t<T, 0<x<1, \\
& u(t, 0)=u(t, 1)=0, \quad 0<t<T, \\
& u(0, x)=u_{0}(x), \quad 0<x<1,
\end{aligned}
$$

where the Riesz fractional derivative is defined by, [31], [32]

$$
\frac{\partial^{2 \alpha} w(x)}{\partial|x|^{2 \alpha}}=-\frac{1}{2 \cos (\alpha \pi)}\left({ }_{0}^{R} D_{x}^{2 \alpha} w(x)+{ }_{x}^{R} D_{1}^{2 \alpha} w(x)\right),
$$

and ${ }_{0}^{R} D_{x}^{\gamma} w(x)$ and ${ }_{x}^{R} D_{1}^{\gamma} w(x), 1<\gamma<2$ are called the left-sided and right-sided Riemann-Liouville fractional derivatives, respectively,

$$
{ }_{0}^{R} D_{x}^{\gamma} w(x)=\frac{1}{\Gamma(2-\gamma)} \frac{d^{2}}{d x^{2}} \int_{0}^{x}(x-y)^{1-\gamma} w(y) d y, \quad{ }_{x}^{R} D_{1}^{\gamma} w(x)=\frac{1}{\Gamma(2-\gamma)} \frac{d^{2}}{d x^{2}} \int_{x}^{1}(y-x)^{1-\gamma} w(y) d y .
$$

\footnotetext{
${ }^{*}$ Corresponding author Khan)

Email addresses: 78525779@qq.com (Yanmei Liu), y.yan@chester.ac.uk (Yubin Yan), sohel_ban@yahoo.com (Monzorul 
Space fractional partial differential equations are widely used to model complex phenomena, for example, in quasi-geostrophic flow, the fast rotating fluids, the dynamic of the frontogenesis in meteorology, the diffusions in fractal or disordered medium, the pollution problems, the mathematical finance and the transport problems, soil contamination and underground water flow, see, e.g., [5], [9], [24], [4], [29].

In recent years, many authors consider the numerical methods for solving space fractional partial differential equations, e.g., finite difference methods [1], [2], [27]-[28], [34]-[38], [40], [19], [30], finite element methods [11], [12]-[18], [33], [41], [42] and spectral methods [25]-[26], [7], [8], matrix transfer technique (MTT) [20]-[21]. Recently, Jin et al. [23] considered the finite element method for solving the linear space fractional parabolic equation where the space fractional derivative is defined as left-sided Riemann-Liouville derivative, see also [22]. The estimates in [23] are for both smooth and nonsmooth initial data, and are expressed directly in terms of the smoothness of the initial data.

The Riesz space fractional partial differential equations were firstly proposed by Chaves [10] to investigate the mechanism of super-diffusion. Benson et al. [3] [4] considered the fractional order governing equation of Lévy motion. Zhang et al. [41] considered a finite element method in space and backward difference method in time for solving Riesz space fractional partial differential equation. Sousa [36] studied a second order numerical method for Riesz space fractional convection-diffusion equation. Bu et al. [6] considered a finite element method in space and Crank-Nicolson method in time for solving Riesz space fractional partial differential equations in two-dimensional case. Duan et al. [13] studied a finite element method in space and backward Euler method in time for solving Riesz space fractional partial differential equations in two-dimensional case.

In this paper, we will consider a finite element method in space and a discontinuous Galerkin method in time for solving Riesz space fractional partial differential equation. When the approximating functions are piecewise constant in time, we proved that the error is $O\left(h^{r-\alpha}+k_{n}\right)$ and the bounds contain the terms $\|u\|_{r, J_{n}}$ and $\left\|u_{t}\right\|_{\alpha, J_{n}}$, see Theorem 4.1 below. When the approximating functions are piecewise linear in time, we proved that the error is $O\left(h^{2(r-\alpha)}+k_{n}^{3}\right)$ and the bounds contain the terms $\|u\|_{r, J_{n}}$ and $\left\|u_{t t}\right\|_{r, J_{n}}$, see Theorem 4.3 below. The advantages of the discontinuous Galerkin method is that, e.g., variable coefficients and nonlinearities present no complication in principle. We obtain precise error estimates for the discontinuous Galerkin method which make it possible to construct the adaptive methods based on the automatic time-step control.

The paper is organized as follows. In Section 2, we introduce some fractional Sobolev spaces and some basic lemmas. In Section 3, we give the error estimates for the backward Euler method. In Section 4, we consider the error estimates for the discontinuous Galerkin time stepping method for $q=1,2$. Finally in Section 4 , we give two numerical examples.

By $C$ we denote a positive constant independent of the functions and parameters concerned, but not necessarily the same at different occurrences.

\section{Preliminaries}

In this section, we will introduce some fractional Sobolev spaces.

Definition 2.1. [14], [25] For any $\sigma>0$, we define the spaces ${ }^{l} H_{0}^{\sigma}(0,1)$ and ${ }^{r} H_{0}^{\sigma}(0,1)$ to be the closures of $C_{0}^{\infty}(0,1)$ with respect to the norms $\|v\|_{{ }^{\imath} H_{0}^{\sigma}(0,1)}$ and $\|v\|_{{ }^{r} H_{0}^{\sigma}(0,1)}$, respectively, where

$$
\|v\|_{{ }^{H} H_{0}^{\sigma}(0,1)}^{2}:=\|v\|_{L^{2}(0,1)}^{2}+\left\|{ }_{0}^{R} D_{x}^{\sigma} v\right\|_{L^{2}(0,1)}^{2},
$$

and

$$
\|v\|_{{ }_{r} H_{0}^{\sigma}(0,1)}^{2}:=\|v\|_{L^{2}(0,1)}^{2}+\left\|{ }_{x}^{R} D_{1}^{\sigma} v\right\|_{L^{2}(0,1)}^{2} .
$$

The semi-norms are defined by $|v|_{{ }^{\imath} H_{0}^{\sigma}(0,1)}:=\left\|{ }_{0}^{R} D_{x}^{\sigma} v\right\|_{L^{2}(0,1)}$ and $|v|_{{ }^{r} H_{0}^{\sigma}(0,1)}:=\left\|{ }_{x}^{R} D_{1}^{\sigma} v\right\|_{L^{2}(0,1)}$, respectively. 
Remark 2.1. In Definition 2.1, $|v|^{\imath}{ }_{{ }_{0}}^{\sigma}(0,1), \sigma>0$ is a semi-norm (not a norm) since $|v|{ }^{\imath} H_{0}^{\sigma}(0,1)=0$ does not imply $v=0$. For example, when $0<\sigma<1$, let $w(x)=x^{\sigma-1}$, we have $w(x) \neq 0$ and

$$
\begin{aligned}
{ }_{0}^{R} D_{x}^{\sigma} w(x) & =\frac{1}{\Gamma(1-\sigma)} \frac{d}{d x} \int_{0}^{x}(x-y)^{-\sigma} w(y) d y=\frac{1}{\Gamma(1-\sigma)} \frac{d}{d x} \int_{0}^{x}(x-y)^{-\sigma} y^{\sigma-1} d y \\
& =\frac{1}{\Gamma(1-\sigma)} \frac{d}{d x} \int_{0}^{1} t^{-\sigma}(1-t)^{\sigma-1} d t=0
\end{aligned}
$$

which implies that $|w|_{{ }^{\imath} H_{0}^{\sigma}(0,1)}=\left\|{ }_{0}^{R} D_{x}^{\sigma} w\right\|_{L^{2}(0,1)}=0$. The similar comments are for the semi-norm $|v|{ }^{r} H_{0}^{\sigma}(0,1)$ and the semi-norm in Definitions 2.2 below.

Definition 2.2. [14], [25] For any $\sigma>0, \sigma \neq n-1 / 2, n \in \mathbb{Z}^{+}$, we define the space ${ }^{c} H_{0}^{\sigma}(0,1)$ to be the closure of $C_{0}^{\infty}(0,1)$ with respect to the norm $\|v\|^{c} H_{0}^{\sigma}(0,1)$, where

$$
\|v\|_{{ }^{c} H_{0}^{\sigma}(0,1)}^{2}:=\|v\|_{L^{2}(0,1)}^{2}+\left|\left({ }_{0}^{R} D_{x}^{\sigma} v,{ }_{x}^{R} D_{1}^{\sigma} v\right)\right|
$$

The semi-norm is defined by $|v|_{c}^{2} H_{0}^{\sigma}(0,1):=\left|\left({ }_{0}^{R} D_{x}^{\sigma} v,{ }_{x}^{R} D_{1}^{\sigma} v\right)\right|$.

Definition 2.3. [14], [25] For any $\sigma>0$, let $H^{\sigma}(\mathbb{R})$ denote the fractional Sobolev space defined in the whole line $\mathbb{R}$. We define

$$
H^{\sigma}(0,1)=\left\{v \in L^{2}(0,1):\left.\tilde{v}\right|_{(0,1)}=v, \text { where } \tilde{v} \in H^{\sigma}(\mathbb{R})\right\}
$$

with the norm

$$
\|v\|_{H^{\sigma}(0,1)}=\inf _{\tilde{v} \in H^{\sigma}(\mathbb{R}),\left.\tilde{v}\right|_{(0,1)}=v}\|\tilde{v}\|_{H^{\sigma}(\mathbb{R})}
$$

where

$$
\|\tilde{v}\|_{H^{\sigma}(\mathbb{R})}=\left\|\left(1+|w|^{2}\right)^{\sigma / 2} \mathcal{F}(\tilde{v})(w)\right\|_{L^{2}(\mathbb{R})},
$$

and $\mathcal{F}(\tilde{v})$ denotes the Fourier transform of $\tilde{v}$ and the corresponding semi-norm is defined by $|\tilde{v}|_{H^{\sigma}(\mathbb{R})}=$ $\left\||w|^{\sigma} \mathcal{F}(\tilde{v})\right\|_{L^{2}(\mathbb{R})}$. Further we define the Sobolev space $H_{0}^{\sigma}(0,1)$ to be the closure of $C_{0}^{\infty}(0,1)$ with respect to the norm $\|v\|_{H^{\sigma}(0,1)}$ and the semi-norm in $H_{0}^{\sigma}(0,1)$ is denoted by $|v|_{H_{0}^{\sigma}(0,1)}$.

Lemma 2.1. [14, Theorems 2.12, 2.13], [25, Lemmas 2.4, 2.5] Let $\sigma>0, \sigma \neq n-1 / 2, n \in \mathbb{Z}^{+}$. The semi-norms and norms in spaces ${ }^{l} H_{0}^{\sigma}(0,1),{ }^{r} H_{0}^{\sigma}(0,1),{ }^{c} H_{0}^{\sigma}(0,1)$ and $H_{0}^{\sigma}(0,1)$ are equivalent.

Below we will denote $(\cdot, \cdot)$ and $\|\cdot\|$ as the inner product and norm in $L^{2}(0,1)$, respectively.

Lemma 2.2. Let $\sigma>0, \sigma \neq n-1 / 2, n \in \mathbb{Z}^{+}$, we have

$$
\left({ }_{0}^{R} D_{x}^{\sigma} v,{ }_{x}^{R} D_{1}^{\sigma} v\right)=\cos (\pi \sigma)\left\|_{0}^{R} D_{x}^{\sigma} v\right\|^{2}, \forall v \in H_{0}^{\sigma}(0,1) .
$$

In particular, $\left({ }_{0}^{R} D_{x}^{\sigma} v,{ }_{x}^{R} D_{1}^{\sigma} v\right)$ is negative when $1 / 2<\sigma \leq 1$.

Proof: It is sufficient to prove

$$
\left({ }_{0}^{R} D_{x}^{\sigma} \varphi,{ }_{x}^{R} D_{1}^{\sigma} \varphi\right)=\cos (\pi \sigma)\left\|_{0}^{R} D_{x}^{\sigma} \varphi\right\|^{2}, \forall \varphi \in C_{0}^{\infty}(0,1) .
$$

In fact, we have, for any $\varphi \in C_{0}^{\infty}(0,1),[25]$,

$$
\left({ }_{0}^{R} D_{x}^{\sigma} \varphi,{ }_{x}^{R} D_{1}^{\sigma} \varphi\right)=\left({ }_{-\infty}^{R} D_{x}^{\sigma} \tilde{\varphi},{ }_{x}^{R} D_{\infty}^{\sigma} \tilde{\varphi}\right)_{L^{2}(\mathbb{R})}=\cos (\pi \sigma)\left\|_{-\infty}^{R} D_{x}^{\sigma} \tilde{\varphi}\right\|_{L^{2}(\mathbb{R})}^{2}=\cos (\pi \sigma)\left\|_{0}^{R} D_{x}^{\sigma} \varphi\right\|^{2},
$$

where $\tilde{\varphi}$ is the extension of $\varphi$ by zero outside of $(0,1)$. 
Lemma 2.3. Let $1 / 2<\alpha \leq 1$. We have, see [25],

$$
\begin{aligned}
& \left({ }_{0}^{R} D_{x}^{2 \alpha} w, v\right)=\left({ }_{0}^{R} D_{x}^{\alpha} w,{ }_{x}^{R} D_{1}^{\alpha} v\right), \forall w, v \in H_{0}^{\alpha}(0,1), \\
& \left({ }_{x}^{R} D_{1}^{2 \alpha} w, v\right)=\left({ }_{x}^{R} D_{1}^{\alpha} w,{ }_{0}^{R} D_{x}^{\alpha} v\right), \forall w, v \in H_{0}^{\alpha}(0,1) .
\end{aligned}
$$

We also have the following fractional Poincaré inequality:

Lemma 2.4. [14], [17], [25] For $u \in H_{0}^{\alpha}(0,1), 1 / 2<\alpha \leq 1$, we have

$$
\|u\|_{L^{2}(0,1)} \leq C|u|_{H_{0}^{\alpha}(0,1)},
$$

and for $0<s<\mu, s \neq n-1 / 2, n \in \mathbb{Z}^{+}$,

$$
|u|_{H_{0}^{s}(0,1)} \leq C|u|_{H_{0}^{\mu}(0,1)} .
$$

Multiplying $v \in H_{0}^{\alpha}(0,1)$ in both sides of the equation (1) and integrating on $(0,1)$ we get, by Lemma 2.3

$$
\begin{aligned}
& \left(u_{t}, v\right)+B_{\alpha}(u, v)=(f, v), \quad \forall v \in H_{0}^{\alpha}(0,1), \\
& u(0)=u_{0},
\end{aligned}
$$

where the bilinear form $B_{\alpha}(\cdot, \cdot)$ is defined by

$$
B_{\alpha}(u, v)=\frac{1}{2 \cos (\alpha \pi)}\left(\left({ }_{0}^{R} D_{x}^{\alpha} u,{ }_{x}^{R} D_{1}^{\alpha} v\right)+\left({ }_{x}^{R} D_{1}^{\alpha} u,{ }_{0}^{R} D_{x}^{\alpha} v\right)\right) .
$$

By Lemmas 2.1, 2.2 and 2.4, it is easy to show that the bilinear form $B_{\alpha}(\cdot, \cdot)$ is symmetric, continuous and coercive on $H_{0}^{\alpha}(0,1), 1 / 2<\alpha \leq 1$.

Let $S_{h} \subset H_{0}^{\alpha}(0,1), 1 / 2<\alpha \leq 1$ be the piecewise continuous linear finite element space. The finite element method of (1)-(3) is to find $u_{h}(t) \in S_{h}$ such that

$$
\begin{aligned}
& \left(u_{h, t}, \chi\right)+B_{\alpha}\left(u_{h}, \chi\right)=(f, \chi), \quad \forall \chi \in S_{h}, \\
& u_{h}(0)=v_{h},
\end{aligned}
$$

where $v_{h} \in S_{h}$ is some appropriate approximation of $u_{0} \in L^{2}(0,1)$.

\section{The backward Euler method}

In this section, we will consider the error estimates of the backward Euler method for solving (5)-(6). Let us first consider the error estimates for solving (5)-(6) in the semidiscrete case.

To do this, we need to introduce the regularity assumption for the following fractional elliptic problem, with $1 / 2<\alpha \leq 1, g \in L^{2}(0,1)$,

$$
\begin{aligned}
& -\frac{\partial^{2 \alpha} w(x)}{\partial|x|^{2 \alpha}}=\frac{1}{2 \cos (\alpha \pi)}\left({ }_{0}^{R} D_{x}^{2 \alpha} w(x)+{ }_{x}^{R} D_{1}^{2 \alpha} w(x)\right)=g(x), 0<x<1, \\
& w(0)=w(1)=0 .
\end{aligned}
$$

The variational form of (10)-(11) is to find $w \in H_{0}^{\alpha}(0,1)$ such that

$$
B_{\alpha}(w, \varphi)=(g, \varphi), \quad \forall \varphi \in H_{0}^{\alpha}(0,1) .
$$


Assumption 3.1. Let $1 / 2<\alpha \leq 1$. For $w$ solving (12) with $g \in L^{2}(0,1)$, there exists some $r \in[\alpha, 2 \alpha]$, such that

$$
\|w\|_{H_{0}^{r}(0,1)} \leq C\|g\|_{L^{2}(0,1)} .
$$

Remark 3.1. Suppose that the equation (10) only contain the left-sided Riemann-Liouville derivative, Jin et al. [23, Lemma 4.2] and [22, Theorem 4.4] show that $r=2 \alpha-1+\beta, 0 \leq \beta<1 / 2$ for $1 / 2<\alpha \leq 1$ in the Assumption 3.1. For the equation (12) with the Riesz fractional derivative, we have at least $w \in H_{0}^{\alpha}(0,1)$. Further we assume that, by the Assumption 3.1, there exists $r \in[\alpha, 2 \alpha]$ such that $w \in H^{r}(0,1) \cap H_{0}^{\alpha}(0,1)$. The similar assumption was also used in [14, Assumption 4.1].

We next introduce the fractional Ritz projection $R_{h, \alpha}$ on $S_{h}$.

Definition 3.1. Let $1 / 2<\alpha \leq 1$ and let $v \in H_{0}^{\alpha}(0,1)$. We define $R_{h, \alpha}: H_{0}^{\alpha}(0,1) \rightarrow S_{h}$ by

$$
B_{\alpha}\left(R_{h, \alpha} v, \chi\right)=B_{\alpha}(v, \chi), \quad \forall \chi \in S_{h}, \quad v \in H_{0}^{\alpha}(0,1) .
$$

It is easy to see that $R_{h, \alpha}: H_{0}^{\alpha}(0,1) \rightarrow S_{h}$ is well defined since $B_{\alpha}(\cdot, \cdot)$ is symmetric, continuous and coercive on $S_{h}$. Further we have, see [14],

Lemma 3.2. Let $v \in H^{r}(0,1) \cap H_{0}^{\alpha}(0,1), 1 / 2<\alpha \leq 1, \alpha \leq r \leq 2 \alpha$ and let $R_{h, \alpha}: H_{0}^{\alpha}(0,1) \rightarrow S_{h}$ be the fractional Ritz projection onto $S_{h}$ defined as in (13). Then, under Assumption 3.1, there exists a constant $C=C(\alpha)$ such that

$$
\left\|R_{h, \alpha} v-v\right\|+h^{r-\alpha}\left|R_{h, \alpha} v-v\right|_{H_{0}^{\alpha}(0,1)} \leq C h^{2(r-\alpha)}\|v\|_{H^{r}(0,1)} .
$$

Theorem 3.3. Let $u_{h}$ and $u$ be the solutions of (8)-(9) and (5)-(6), respectively. Let $\alpha \leq r \leq 2 \alpha, 1 / 2<$ $\alpha \leq 1$. Let $u_{0} \in H^{r}(0,1)$. Then, under the Assumption 3.1, there exists a constant $C=C(\alpha)$ such that

$$
\left\|u_{h}(t)-u(t)\right\| \leq\left\|v_{h}-u_{0}\right\|+C h^{2(r-\alpha)}\left(\left\|u_{0}\right\|_{H^{r}(0,1)}+\int_{0}^{t}\left\|u_{t}(s)\right\|_{H^{r}(0,1)} d s\right) .
$$

Proof: We write

$$
u_{h}(t)-u(t)=\theta(t)+\rho(t),
$$

where $\theta(t)=u_{h}(t)-R_{h, \alpha} u(t)$ and $\rho(t)=R_{h, \alpha} u(t)-u(t)$.

By Lemma 3.2, we have, with $1 / 2<\alpha \leq 1$,

$$
\|\rho(t)\| \leq C h^{2(r-\alpha)}\|u(t)\|_{H^{r}(0,1)} .
$$

Note that

$$
u(t)=u(0)+\int_{0}^{t} u_{t}(s) d s
$$

we get

$$
\|u(t)\|_{H^{r}(0,1)} \leq\left\|u_{0}\right\|_{H^{r}(0,1)}+\int_{0}^{t}\left\|u_{t}(s)\right\|_{H^{r}(0,1)} d s .
$$

Hence

$$
\|\rho(t)\| \leq C h^{2(r-\alpha)}\left(\left\|u_{0}\right\|_{H^{r}(0,1)}+\int_{0}^{t}\left\|u_{t}(s)\right\|_{H^{r}(0,1)} d s\right) .
$$

We next consider the estimates for $\theta(t)$. Note that $\theta(t)$ satisfies

$$
\begin{aligned}
\left(\theta_{t}, \chi\right)+B_{\alpha}(\theta, \chi) & =\left(u_{h, t}, \chi\right)+B_{\alpha}\left(u_{h}, \chi\right)-\left(R_{h, \alpha} u_{t}, \chi\right)-B_{\alpha}(u, \chi) \\
& =(f, \chi)-\left(R_{h, \alpha} u_{t}, \chi\right)-B_{\alpha}(u, \chi)=\left(u_{t}-R_{h, \alpha} u_{t}, \chi\right) \\
& =\left(-\rho_{t}, \chi\right), \quad \forall \chi \in S_{h} .
\end{aligned}
$$


Choose $\chi=\theta$, we get

$$
\left(\theta_{t}, \theta\right)+B_{\alpha}(\theta, \theta)=-\left(\rho_{t}, \theta\right)
$$

which implies, by Lemma 2.1,

$$
\frac{1}{2} \frac{d}{d t}\|\theta\|^{2}+C|\theta|_{H_{0}^{\alpha}(0,1)}^{2} \leq-\left(\rho_{t}, \theta\right) \leq\left\|\rho_{t}\right\|\|\theta\| .
$$

Note that $|\theta|_{H_{0}^{\alpha}(0,1)}^{2}>0$, we get

$$
\frac{1}{2} \frac{d}{d t}\|\theta\|^{2} \leq-\left(\rho_{t}, \theta\right) \leq\left\|\rho_{t}\right\|\|\theta\|
$$

which implies that

$$
\frac{d}{d t}\|\theta(t)\| \leq\left\|\rho_{t}(t)\right\|
$$

Hence,

$$
\begin{aligned}
\|\theta(t)\| & \leq\|\theta(0)\|+\int_{0}^{t}\left\|\rho_{t}(s)\right\| d s \leq\left\|u_{h}(0)-R_{h, \alpha} u(0)\right\|+\int_{0}^{t} C h^{2(r-\alpha)}\left\|u_{t}(s)\right\|_{H^{r}(0,1)} d s \\
& \leq\left\|u_{h}(0)-u(0)\right\|+C h^{2(r-\alpha)}\|u(0)\|_{H^{r}(0,1)}+\int_{0}^{t} C h^{2(r-\alpha)}\left\|u_{t}(s)\right\|_{H^{r}(0,1)} d s .
\end{aligned}
$$

Together these estimates complete the proof of Theorem 3.3 .

We now introduce the backward Euler methods for solving (5)-(6). Let $0=t_{0}<t_{1}<t_{2}<\cdots<t_{N}=T$ be a partition of $[0, T]$ and $k$ be the time step size. Let $U^{n} \approx u_{h}\left(t_{n}\right)$ be the approximation of $u_{h}\left(t_{n}\right)$. The backward Euler method for solving (5)-(6) is to find $U^{n} \in S_{h}$, such that

$$
\begin{aligned}
& \left(\frac{U^{n}-U^{n-1}}{k}, \chi\right)+B_{\alpha}\left(U^{n}, \chi\right)=\left(f\left(t_{n}\right), \chi\right), \forall \chi \in S_{h}, \\
& U^{0}=v_{h},
\end{aligned}
$$

or

$$
\begin{aligned}
& \left(U^{n}, \chi\right)+k B_{\alpha}\left(U^{n}, \chi\right)=\left(U^{n-1}+k f\left(t_{n}\right), \chi\right), \\
& U^{0}=v_{h} .
\end{aligned}
$$

Theorem 3.4. Let $U^{n}$ and $u\left(t_{n}\right)$ be the solutions of (16)-(17) and (5)-(6), respectively. Let $\alpha \leq r \leq$ $2 \alpha, 1 / 2<\alpha \leq 1$. Assume that $u_{0} \in H^{r}(0,1)$ and

$$
\left\|v_{h}-u_{0}\right\| \leq C h^{2(r-\alpha)}\left\|u_{0}\right\|_{H^{r}(0,1)} .
$$

We have, under the Assumption 3.1, with $n=1,2, \ldots, N$,

$$
\left\|U^{n}-u\left(t_{n}\right)\right\| \leq C h^{2(r-\alpha)}\left(\left\|u_{0}\right\|_{H^{r}(0,1)}+\int_{0}^{t_{n}}\left\|u_{t}\right\|_{H^{r}(0,1)} d s\right)+k \int_{0}^{t_{n}}\left\|u_{t t}\right\| d s .
$$

Proof: We write

$$
U^{n}-u\left(t_{n}\right)=\left(U^{n}-R_{h, \alpha} u\left(t_{n}\right)\right)+\left(R_{h, \alpha} u\left(t_{n}\right)-u\left(t_{n}\right)\right)=\theta^{n}+\rho^{n} .
$$


Here $\rho^{n}=\rho\left(t_{n}\right)$ is bounded by

$$
\begin{aligned}
\left\|\rho^{n}\right\| & =\left\|R_{h, \alpha} u\left(t_{n}\right)-u\left(t_{n}\right)\right\| \leq C h^{2(r-\alpha)}\left\|u\left(t_{n}\right)\right\|_{H^{r}(0,1)} \leq C h^{2(r-\alpha)}\left\|u_{0}+\int_{0}^{t} u_{t}(s) d s\right\|_{H^{r}(0,1)} \\
& \leq C h^{2(r-\alpha)}\left(\left\|u_{0}\right\|_{H^{r}(0,1)}+\int_{0}^{t}\left\|u_{t}\right\|_{H^{r}(0,1)} d s\right) .
\end{aligned}
$$

We next estimate $\theta^{n}$. Note that $\theta^{n}$ satisfies, by (16)-(17) and (5)-(6),

$$
\begin{aligned}
& \left(\frac{\theta^{n}-\theta^{n-1}}{k}, \chi\right)+B_{\alpha}\left(\theta^{n}, \nabla \chi\right) \\
& =\left(\frac{U^{n}-U^{n-1}}{k}, \chi\right)-\left(R_{h, \alpha} \frac{u\left(t_{n}\right)-u\left(t_{n-1}\right)}{k}, \chi\right)+B_{\alpha}\left(U^{n}, \chi\right)-B_{\alpha}\left(R_{h, \alpha} u\left(t_{n}\right), \chi\right) \\
& =\left(u_{t}\left(t_{n}\right), \chi\right)-\left(R_{h, \alpha} \frac{u\left(t_{n}\right)-u\left(t_{n-1}\right)}{k}, \chi\right) \\
& =\left(u_{t}\left(t_{n}\right)-\frac{u\left(t_{n}\right)-u\left(t_{n-1}\right)}{k}, \chi\right)+\left(\frac{u\left(t_{n}\right)-u\left(t_{n-1}\right)}{k}-R_{h, \alpha} \frac{u\left(t_{n}\right)-u\left(t_{n-1}\right)}{k}, \chi\right) \\
& =-\left(w^{n}, \chi\right)
\end{aligned}
$$

where $w^{n}=w_{1}^{n}+w_{2}^{n}$,

$$
w_{1}^{n}=\left(R_{h, \alpha}-I\right) \frac{u\left(t_{n}\right)-u\left(t_{n-1}\right)}{k}, \quad w_{2}^{n}=\frac{u\left(t_{n}\right)-u\left(t_{n-1}\right)}{k}-u_{t}\left(t_{n}\right) .
$$

Choose $\chi=\theta^{n}$ in (20), we have

$$
\left(\theta^{n}, \theta^{n}\right)-\left(\theta^{n-1}, \theta^{n}\right)+k B_{\alpha}\left(\theta^{n}, \theta^{n}\right)=-k\left(w^{n}, \theta^{n}\right) .
$$

Note that, by the coercivity property, $B_{\alpha}\left(\theta^{n}, \theta^{n}\right) \geq 0$, we have

$$
\left\|\theta^{n}\right\|^{2}-\left(\theta^{n-1}, \theta^{n}\right) \leq k\left\|w^{n}\right\|\left\|\theta^{n}\right\|,
$$

or

$$
\left\|\theta^{n}\right\|^{2} \leq\left\|\theta^{n-1}\right\|\left\|\theta^{n}\right\|+k\left\|w^{n}\right\|\left\|\theta^{n}\right\| .
$$

Cancelling $\left\|\theta^{n}\right\|$, we get

$$
\left\|\theta^{n}\right\| \leq\left\|\theta^{n-1}\right\|+k\left\|w^{n}\right\| .
$$

By repeated application, we have

$$
\left\|\theta^{n}\right\| \leq\|\theta(0)\|+k \sum_{j=1}^{n}\left\|w^{j}\right\| \leq\|\theta(0)\|+k \sum_{j=1}^{n}\left\|w_{1}^{j}\right\|+k \sum_{j=1}^{n}\left\|w_{2}^{j}\right\| .
$$

We write

$$
\begin{aligned}
w_{1}^{j} & =\left(R_{h, \alpha}-I\right) \frac{u\left(t_{j}\right)-u\left(t_{j-1}\right)}{k} \\
& =\left(R_{h, \alpha}-I\right) k^{-1} \int_{t_{j-1}}^{t_{j}} u_{t}(s) d s=k^{-1} \int_{t_{j-1}}^{t_{j}}\left(R_{h, \alpha}-I\right) u_{t}(s) d s
\end{aligned}
$$

Hence

$$
\begin{aligned}
k \sum_{j=1}^{n}\left\|w_{1}^{j}\right\| & \leq \sum_{j=1}^{n} \int_{t_{j-1}}^{t_{j}}\left\|\left(R_{h, \alpha}-I\right) u_{t}(s)\right\| d s \\
& \leq \sum_{j=1}^{n} \int_{t_{j-1}}^{t_{j}} C h^{2(r-\alpha)}\left\|u_{t}\right\|_{H^{r}(0,1)} d s \leq C h^{2(r-\alpha)} \int_{0}^{t_{n}}\left\|u_{t}\right\|_{H^{r}(0,1)} d s .
\end{aligned}
$$


Further

$$
k w_{2}^{j}=u\left(t_{j}\right)-u\left(t_{j-1}\right)-k u_{t}\left(t_{j}\right)=-\int_{t_{j-1}}^{t_{j}}\left(s-t_{j-1}\right) u_{t t}(s) d s .
$$

Thus

$$
k \sum_{j=1}^{n}\left\|w_{2}^{j}\right\| \leq \sum_{j=1}^{n}\left\|\int_{t_{j-1}}^{t_{j}}\left(s-t_{j-1}\right) u_{t t}(s) d s\right\| \leq k \sum_{j=1}^{n} \int_{t_{j-1}}^{t_{j}}\left\|u_{t t}(s)\right\| d s=k \int_{0}^{t_{n}}\left\|u_{t t}(s)\right\| d s .
$$

Together these estimates complete the proof of Theorem 3.4.

\section{The discontinuous Galerkin time stepping method}

In Section 3, we obtain the error estimates for solving (5)-(6) by using the finite element method in space and the backward Euler method in time. The error is $O\left(h^{2(r-\alpha)}+k\right), \alpha \leq r \leq 2 \alpha, 1 / 2<\alpha \leq 1$ and the bounds contain the terms $\int_{0}^{t_{n}}\left\|u_{t}(s)\right\|_{H^{r}(0,1)} d s$ and $\int_{0}^{t_{n}}\left\|u_{t t}(s)\right\| d s$. In this section, we will consider the discontinuous Galerkin time stepping method for solving (5)-(6). When the approximating functions are piecewise constant in time, we proved that the error is $O\left(h^{r-\alpha}+k_{n}\right)$ and the bounds contain the terms $\|u\|_{r, J_{n}}$ and $\left\|u_{t}\right\|_{\alpha, J_{n}}$, see Theorem 4.1 below. When the approximating functions are piecewise linear in time, we proved that the error is $O\left(h^{2(r-\alpha)}+k_{n}^{3}\right)$ and the bounds contain the terms $\|u\|_{r, J_{n}}$ and $\left\|u_{t t}\right\|_{\alpha, J_{n}}$, see Theorem 4.3 below.

Let $0=t_{0}<t_{1}<\cdots<t_{n-1}<t_{n}<\cdots<t_{N}=T$ be the time partition of $[0, T]$. Let $k_{n}=t_{n}-t_{n-1}, n=$ $1,2, \ldots, N$ be the time step size. Denote $J_{n}=\left(t_{n-1}, t_{n}\right]$.

Define

$$
S_{k h}=\left\{X:[0, T] \rightarrow S_{h},\left.X\right|_{J_{n}}=\sum_{j=0}^{q-1} X_{j} t^{j}, X_{j} \in S_{h}\right\}
$$

where $q$ is a given positive integer and $X=X(t) \in S_{k h}$ is left continuous at the discretization point $t_{n}$, but not necessarily right continuous at $t_{n-1}$ on each subinterval $J_{n}=\left(t_{n-1}, t_{n}\right], n=1,2, \ldots, N$. Denote $X_{-}^{n}=$ $X\left(t_{n}-\right)=\lim _{t \rightarrow t_{n}-} X(t)$ and $X_{+}^{n-1}=X\left(t_{n-1}+\right)=\lim _{t \rightarrow t_{n-1}+} X(t)$. We then have $X_{-}^{n}=X\left(t_{n}\right)=X^{n}$. Further, let $S_{k h}^{n}$ denote the restriction of $S_{k h}$ on $J_{n}=\left(t_{n-1}, t_{n}\right]$.

The discontinuous Galerkin time stepping method of (5)-(6) is to find $U=U(t) \in S_{k h}^{n}$ such that, with $n=1,2, \ldots, N$,

$$
\begin{gathered}
\int_{t_{n-1}}^{t_{n}}\left[\left(U_{t}, X\right)+B_{\alpha}(U, X)\right] d t+\left(U_{+}^{n-1}, X_{+}^{n-1}\right) \\
=\left(U_{-}^{n-1}, X_{+}^{n-1}\right)+\int_{t_{n-1}}^{t_{n}}(f, X) d t, \forall X \in S_{k h}^{n}, \\
U\left(t_{n-1}\right)=U_{-}^{n-1},
\end{gathered}
$$

or

$$
\begin{gathered}
\int_{t_{0}}^{t_{N}}\left[\left(U_{t}, X\right)+B_{\alpha}(U, X)\right] d t+\sum_{n=1}^{N-1}\left([U]_{n}, X_{+}^{n}\right)+\left(U_{+}^{0}, X_{+}^{0}\right) \\
=\left(U_{-}^{0}, X_{+}^{0}\right)+\int_{t_{0}}^{t_{N}}(f, X) d t, \forall X \in S_{k h}, \\
U(0)=U_{-}^{0}=v_{h} .
\end{gathered}
$$


Here $[U]_{n}=U_{+}^{n}-U_{-}^{n}$ denotes the jump of $U$ at $t_{n}, n=1,2, \ldots, N-1$.

Denote

$$
\bar{B}_{\alpha}(U, X)=\int_{t_{0}}^{t_{N}}\left[\left(U_{t}, X\right)+B_{\alpha}(U, X)\right] d t+\sum_{n=1}^{N-1}\left([U]_{n}, X_{+}^{n}\right)+\left(U_{+}^{0}, X_{+}^{0}\right) .
$$

Then the discontinuous Galerkin time stepping method of (5)-(6) is to find $U \in S_{k h}$ such that

$$
\bar{B}_{\alpha}(U, X)=\left(U_{-}^{0}, X_{+}^{0}\right)+\int_{t_{0}}^{t_{N}}(f, X) d t, \quad \forall X \in S_{k h} .
$$

We remark that in the case $q=1,(21)$-(22) reduces to the following modified backward Euler method

$$
\left(U^{n}, \chi\right)+k_{n} B_{\alpha}\left(U^{n}, \chi\right)=\left(U^{n-1}, \chi\right)+\left(\int_{t_{n-1}}^{t_{n}} f(t) d t, \chi\right), \quad \forall \chi \in S_{h} .
$$

Note that the $f^{n}=f\left(t_{n}\right)$ occurring in the standard backward Euler method (18)-(19) has been replaced by an average of $f$ over $\left(t_{n-1}, t_{n}\right)$. The standard backward Euler method may thus be interpreted as resulting from (26) after quadrature.

We have the following theorem.

Theorem 4.1. Assume that $k_{n+1} / k_{n} \geq c>0$ for $n \geq 1$ and let $q=1$. Let $U^{n}$ and $u\left(t_{n}\right)$ be the solutions of (21)-(22) and (5)-(6), respectively. Let $\alpha \leq r \leq 2 \alpha, 1 / 2<\alpha \leq 1$. Then we have, under the Assumption 3.1, with $v_{h}=P_{h} u_{0}, u_{0} \in L^{2}(0,1)$,

$$
\left\|U^{N}-u\left(t_{N}\right)\right\| \leq C L_{N} \max _{n \leq N}\left(h^{r-\alpha}\|u\|_{r, J_{n}}+k_{n}\left\|u_{t}\right\|_{\alpha, J_{n}}\right),
$$

where $L_{N}=1+\left(\log \frac{t_{N}}{k_{N}}\right)^{1 / 2}$ and $\|\varphi\|_{s, J_{n}}=\sup _{t \in J_{n}}\|\varphi(t)\|_{H^{s}(0,1)}, s=\alpha, r$.

Denote $A_{\alpha}: \mathcal{D}\left(A_{\alpha}\right) \rightarrow L^{2}(0,1)$ by

$$
B_{\alpha}(\varphi, \psi)=\left(A_{\alpha} \varphi, \psi\right), \quad \forall \varphi \in \mathcal{D}\left(A_{\alpha}\right), \psi \in H_{0}^{\alpha}(0,1) .
$$

We may consider the following backward homogeneous problem

$$
\begin{aligned}
& -z_{t}+A_{\alpha} z=0, \text { for } t<t_{N}, \\
& z\left(t_{N}\right)=\varphi .
\end{aligned}
$$

We next introduce the discrete fractional elliptic operator $A_{h, \alpha}: S_{h} \rightarrow S_{h}$ by, with $1 / 2<\alpha \leq 1$,

$$
\left(A_{h, \alpha} \psi, \chi\right)=\frac{1}{2 \cos (\pi \alpha)}\left[\left({ }_{0}^{R} D_{x}^{\alpha} \psi,{ }_{x}^{R} D_{1}^{\alpha} \chi\right)+\left({ }_{x}^{R} D_{1}^{\alpha} \psi,{ }_{0}^{R} D_{x}^{\alpha} \chi\right)\right], \quad \forall \psi, \chi \in S_{h} .
$$

The semidiscrete problem of (28)-(29) is then to find $z_{h} \in S_{h}$ such that

$$
\begin{aligned}
& -z_{h, t}+A_{h, \alpha} z_{h}=0, \quad \text { for } t<t_{N}, \\
& z_{h}\left(t_{N}\right)=P_{h} \varphi .
\end{aligned}
$$

The discontinuous Galerkin time stepping method for (31)-(32) is to find $Z_{h} \in S_{k h}^{n}$ such that

$$
\begin{gathered}
\int_{t_{n-1}}^{t_{n}}\left[\left(X_{h},-Z_{h, t}\right)+B_{\alpha}\left(X_{h}, A_{h, \alpha} Z_{h}\right)\right] d t+\left(X_{h}\left(t_{n}-\right), Z_{h}\left(t_{n}-\right)\right) \\
=\left(X_{h}\left(t_{n}-\right), Z_{h}\left(t_{n}+\right)\right), \quad \forall X_{h} \in S_{k h}^{n}, \\
Z_{h}\left(t_{N}+\right)=Z_{h}\left(t_{N}\right)=P_{h} \varphi .
\end{gathered}
$$


Here we use the fact that $B_{\alpha}\left(X_{h}, Z_{h}\right)=\left(A_{h, \alpha} X_{h}, Z_{h}\right)=\left(X_{h}, A_{h, \alpha} Z_{h}\right)$.

We remark that (33)-(34) are obtained by transforming (31)-(32) into the forward homogeneous problem and then apply the discontinuous Galerkin time stepping method (21)-(22) to this forward homogeneous problem. In fact, let $t=T-s$, we assume

$$
z_{h}(t)=z_{h}(T-s)=\bar{z}_{h}(s),
$$

which implies that

$$
z_{h, t}=-\bar{z}_{h, s}, \quad z_{h}\left(t_{N}\right)=\bar{z}_{h}(0) .
$$

Here (31)-(32) is equivalent to the following forward homogeneous problem

$$
\begin{aligned}
& \bar{z}_{h, t}+A_{h, \alpha} \bar{z}_{h}=0, \quad \text { for } t \leq t_{N}, \\
& \bar{z}_{h}(0)=P_{h} \varphi .
\end{aligned}
$$

The discontinuous Galerkin time stepping method of (35)-(36) is to find $\bar{Z}_{h} \in S_{k h}^{n}$ such that

$$
\begin{aligned}
& \int_{t_{N}-t_{n}}^{t_{N}-t_{n-1}}\left[\left(\bar{Z}_{h, s}, \bar{X}_{h}\right)+\left(A_{h, \alpha} \bar{Z}_{h}, \bar{X}_{h}\right)\right] d s+\left(\bar{Z}_{h}\left(\left(t_{N}-t_{n}\right)+\right), \bar{X}_{h}\left(\left(t_{N}-t_{n}\right)+\right)\right) \\
= & \left(\bar{Z}_{h}\left(\left(t_{N}-t_{n}\right)-\right), \bar{X}_{h}\left(\left(t_{N}-t_{n}\right)-\right)\right), \quad \forall \bar{X}_{h} \in S_{k h}^{n},
\end{aligned}
$$

which implies that, with $s=t_{N}-t, \bar{Z}_{h}(s)=Z_{h}(t), \bar{Z}_{h, s}(s)=-Z_{h, t}(t)$,

$$
\begin{aligned}
& \int_{t_{n-1}}^{t_{n}}\left[\left(X_{h},-Z_{h, t}\right)+\left(X_{h}, A_{h, \alpha} Z_{h}\right)\right] d t+\left(X_{h}\left(t_{N}-\left(t_{N}-t_{n}\right)+\right), Z_{h}\left(t_{N}-\left(t_{N}-t_{n}\right)+\right)\right) \\
= & \left(X_{h}\left(t_{N}-\left(t_{N}-t_{n}\right)-\right), Z_{h}\left(t_{N}-\left(t_{N}-t_{n}\right)-\right), \quad \forall X_{h} \in S_{k h}^{n},\right.
\end{aligned}
$$

or

$$
\begin{aligned}
& \int_{t_{n-1}}^{t_{n}}\left[\left(X_{h},-Z_{h, t}\right)+\left(X_{h}, A_{h, \alpha} Z_{h}\right)\right] d t+\left(X_{h}\left(t_{n}-\right), Z_{h}\left(t_{n}-\right)\right) \\
= & \left(X_{h}\left(t_{n}-\right), Z_{h}\left(t_{n}+\right)\right), \quad \forall X_{h} \in S_{k h}^{n},
\end{aligned}
$$

which is (33)-(34).

By summation with $n=1,2, \ldots, N$, we get

$$
\begin{aligned}
& \int_{t_{0}}^{t_{N}}\left[\left(X_{h},-Z_{h, t}\right)+\left(X_{h}, A_{h, \alpha} Z_{h}\right)\right] d t-\sum_{n=1}^{N-1}\left(X_{h}\left(t_{n}-\right),\left[Z_{h}\right]_{n}\right)+\left(X_{h}\left(t_{N}-\right), Z_{h}\left(t_{N}-\right)\right) \\
= & \left(X_{h}\left(t_{N}-\right), Z_{h}\left(t_{N}+\right)\right)=\left(X_{h}\left(t_{N}-\right), P_{h} \varphi\right), \quad \forall X_{h} \in S_{k h} .
\end{aligned}
$$

It is easy to show that, by integration by parts with respect to $t$,

$$
\begin{aligned}
\bar{B}_{\alpha}\left(X_{h}, Z_{h}\right)= & \int_{t_{0}}^{t_{N}}\left[\left(X_{h},-Z_{h, t}\right)+\left(X_{h}, A_{h, \alpha} Z_{h}\right)\right] d t \\
& -\sum_{n=1}^{N-1}\left(X_{h}\left(t_{n}-\right),\left[Z_{h}\right]_{n}\right)+\left(X_{h}\left(t_{N}-\right), Z_{h}\left(t_{N}-\right)\right) .
\end{aligned}
$$

Hence we see that the solution $Z_{h} \in S_{k h}$ of (33)- (34) satisfies

$$
\bar{B}_{\alpha}\left(X_{h}, Z_{h}\right)=\left(X_{h}\left(t_{N}-\right), P_{h} \varphi\right)=\left(X_{h}\left(t_{N^{-}}\right), \varphi\right), \quad \forall X_{h} \in S_{k h} .
$$


Lemma 4.2. Assume that $k_{n+1} / k_{n} \geq c>0, n \geq 1$. Then we have for the solution of (37),

$$
\int_{0}^{t_{N}}\left(\left\|Z_{h, t}\right\|+\left\|A_{h, \alpha} Z_{h}\right\|\right) d t+\sum_{n=1}^{N}\left\|\left[Z_{h}\right]_{n}\right\| \leq C L_{N}\|\varphi\|
$$

where $L_{N}=1+\left(\log \frac{t_{N}}{k_{N}}\right)^{1 / 2}$.

Proof: The proof is similar to the proof of [39, Lemma 12.3]. We omit the proof here.

Proof of Theorem 4.1: $\quad$ Let $\tilde{u}$ denote the piecewise constant function (with respect to $t$ ) defined by

$$
\tilde{u}(t)=u\left(t_{n}\right), \quad \text { for } t \in\left(t_{n-1}, t_{n}\right],
$$

we write

$$
e=U-u=\left(U-R_{h, \alpha} \tilde{u}\right)+\left(R_{h, \alpha} \tilde{u}-u\right)=\theta+\rho,
$$

where $R_{h, \alpha}$ is defined by (13).

For $\rho$, we have, noting that $\tilde{u}\left(t_{N}\right)=u\left(t_{N}\right)$,

$$
\left\|\rho^{N}\right\|=\left\|R_{h, \alpha} \tilde{u}\left(t_{N}\right)-u\left(t_{N}\right)\right\|=\left\|R_{h, \alpha} u\left(t_{N}\right)-u\left(t_{N}\right)\right\| \leq C h^{2(r-\alpha)}\|u\|_{H^{r}(0,1)} .
$$

For $\theta$, we have, with $\varphi \in L^{2}(0,1)$, by $(37)$,

$$
\bar{B}_{\alpha}\left(\theta, Z_{h}\right)=\left(\theta^{N}, \varphi\right) .
$$

Thus

$$
\left(\theta^{N}, \varphi\right)=\bar{B}_{\alpha}\left(\theta, Z_{h}\right)=\bar{B}_{\alpha}\left(e-\rho, Z_{h}\right)=\bar{B}_{\alpha}\left(e, Z_{h}\right)-\bar{B}_{\alpha}\left(\rho, Z_{h}\right)
$$

Note that

$$
\bar{B}_{\alpha}\left(e, X_{h}\right)=\bar{B}_{\alpha}\left(U-u, X_{h}\right)=0, \quad \forall X_{h} \in S_{k h} .
$$

In fact, we have, by (25),

$$
\bar{B}_{\alpha}\left(U, X_{h}\right)=\left(U_{-}^{0}, X_{h}(0+)\right)+\int_{t_{0}}^{t_{N}}\left(f, X_{h}\right) d t, \quad \forall X_{h} \in S_{k h} .
$$

Further

$$
\begin{aligned}
\bar{B}_{\alpha}\left(u, X_{h}\right) & =\int_{t_{0}}^{t_{N}}\left[\left(u_{t}, X_{h}\right)+\left(A_{\alpha} u, X_{h}\right)\right] d t+\sum_{n=1}^{N-1}\left([u]_{n}, X_{h}\left(t_{n}+\right)\right)+\left(u_{-}^{0}, X_{h}(0+)\right) \\
& =\int_{t_{0}}^{t_{N}}\left(f, X_{h}\right) d t+\left(u_{-}^{0}, X_{h}(0+)\right) .
\end{aligned}
$$

Thus

$$
\bar{B}_{\alpha}\left(e, X_{h}\right)=\left(U_{-}^{0}-u_{-}^{0}, X_{h}(0+)\right)=\left(P_{h} u_{0}-u_{0}, X_{h}\left(t_{0}+\right)\right)=0 .
$$

Therefore

$$
\left(\theta^{N}, \varphi\right)=-\bar{B}_{\alpha}\left(\rho, Z_{h}\right)=-\sum_{n=1}^{N} \int_{t_{n-1}}^{t_{n}}\left[\left(\rho,-Z_{h, t}\right)+B_{\alpha}\left(\rho, Z_{h}\right)\right]+\sum_{n=1}^{N-1}\left(\rho^{n},\left[Z_{h}\right]_{n}\right)-\left(\rho^{N}, P_{h} \varphi\right) .
$$


Note that

$$
B_{\alpha}\left(\rho, Z_{h}\right)=B_{\alpha}\left(R_{h, \alpha} \rho, Z_{h}\right)=\left(R_{h, \alpha} \rho, A_{h, \alpha} Z_{h}\right),
$$

and $\rho^{n}=0, n=1,2, \ldots, N$, we have

$$
\begin{aligned}
\left(\theta^{N}, \varphi\right) & =-\sum_{n=1}^{N} \int_{t_{n-1}}^{t_{n}}\left(R_{h, \alpha} \rho, A_{h, \alpha} Z_{h}\right) d t+\sum_{n=1}^{N-1}\left(\rho^{n},\left[Z_{h}\right]_{n}\right)-\left(\rho^{N}, P_{h} \varphi\right) \\
& \leq \max _{n \leq N}\left(\|\rho\|_{J_{n}}+\left\|R_{h, \alpha} \rho\right\|_{J_{n}}\right)\left[\int_{0}^{t_{N}}\left\|A_{h, \alpha} Z_{h}\right\| d t+\sum_{n=1}^{N-1}\left\|\left[Z_{h}\right]_{n}\right\|+\|\varphi\|\right] .
\end{aligned}
$$

By (14) with $r=\alpha$, we have

$$
\left\|R_{h, \alpha} \rho\right\|_{J_{n}} \leq\left\|R_{h, \alpha} \rho-\rho\right\|_{J_{n}}+\|\rho\|_{J_{n}} \leq C h^{0}\|\rho\|_{\alpha, J_{n}}+\|\rho\|_{J_{n}} \leq C\|\rho\|_{\alpha, J_{n}} .
$$

We therefore have

$$
\left\|\theta^{N}\right\| \leq C L_{N} \max _{n \leq N}\|\rho\|_{\alpha, J_{n}}
$$

Note that,

$$
\begin{aligned}
\|\rho\|_{\alpha, J_{n}} & =\left\|R_{h, \alpha} \tilde{u}-u\right\|_{\alpha, J_{n}} \leq\left\|\left(R_{h, \alpha}-I\right) \tilde{u}\right\|_{\alpha, J_{n}}+\|\tilde{u}-u\|_{\alpha, J_{n}} \\
& =\left\|\left(R_{h, \alpha}-I\right) u\left(t_{n}\right)\right\|_{\alpha, J_{n}}+\|\tilde{u}-u\|_{\alpha, J_{n}} \leq C h^{r-\alpha}\left\|u\left(t_{n}\right)\right\|_{H^{r}(0,1)}+C k_{n}\left\|u_{t}\right\|_{\alpha, J_{n}} \\
& \leq C h^{r-\alpha}\|u\|_{r, J_{n}}+C k_{n}\left\|u_{t}\right\|_{\alpha, J_{n}}
\end{aligned}
$$

Together these estimates complete the proof of Theorem 4.1.

Remark 4.1. Note that, by (14), the fractional Ritz projection $R_{h, \alpha}$ is not bounded in $L^{2}(0,1)$. Therefore we can not bound $\left\|R_{h, \alpha} \rho\right\|$ by using $\|\rho\|$ in (39) as in the proof of (12.50) in [39, pp. 199]. Therefore we can only prove the convergence order $O\left(h^{r-\alpha}\right)$ for the discontinuous Galerkin time stepping method with $q=1$ for space fractional partial differential equation.

Theorem 4.3. Let $q=2$, and assume that $k_{n+1} / k_{n} \geq c>0$ for $n \geq 1$. Let $U^{n}$ and $u\left(t_{n}\right)$ be the solutions of (21)-(22) and (5)-(6), respectively. Let $\alpha \leq r \leq 2 \alpha, 1 / 2<\alpha \leq 1$. Then we have, under the Assumption 3.1, with $v_{h}=P_{h} u_{0}, u_{0} \in L^{2}(0,1)$,

$$
\left\|U^{N}-u\left(t_{N}\right)\right\| \leq C L_{N} \max _{n \leq N}\left(h^{2(r-\alpha)}\|u\|_{r, J_{n}}+k_{n}^{3}\left\|u_{t t}\right\|_{\alpha, J_{n}}\right)
$$

where $L_{N}=1+\left(\log \frac{t_{N}}{k_{N}}\right)^{1 / 2}$ and $\|\varphi\|_{s, J_{n}}=\sup _{t \in J_{n}}\|\varphi(t)\|_{H^{s}(0,1)}, s=\alpha, r$.

Proof: With $J_{n}=\left(t_{n-1}, t_{n}\right], n \geq 1$ and let $\tilde{u} \in S_{k}$ denote the piecewise linear interpolant defined by

$$
\begin{aligned}
& \tilde{u}\left(t_{n}\right)=u\left(t_{n}\right), n \geq 0, \\
& \int_{J_{n}}(\tilde{u}(t)-u(t)) d t=0, n \geq 1,
\end{aligned}
$$

i.e., $\tilde{u}$ interpolates at the nodal points, and the interpolation error is orthogonal to any constant on $J_{n}$. This interpolant is uniquely defined and the error estimates read as follows, See [39, (12.10), pp. 186]

$$
|\tilde{u}(t)-u(t)|_{H_{0}^{j}(0,1)} \leq C k_{n}^{3} \int_{J_{n}}\left|u_{t t}(s)\right|_{H_{0}^{j}(0,1)}, \text { for } t \in J_{n}, j=0,1
$$


This time we find instead of (38)

$$
\left(\theta^{N}, \varphi\right)=-\sum_{n=1}^{N} \int_{J_{n}}\left(-\left(\rho, Z_{h, t}\right)+B_{\alpha}\left(\rho, Z_{h}\right)\right) d t+\sum_{n=1}^{N-1}\left(\rho^{n},\left[Z_{h}\right]_{n}\right)-\left(\rho^{N}, P_{h} \varphi\right) .
$$

Here we have, using the definition of $\tilde{u}$,

$$
\int_{J_{n}}\left(\rho, Z_{h, t}\right) d t=\int_{J_{n}}\left(R_{h, \alpha} \tilde{u}-u, Z_{h, t}\right) d t=\int_{J_{n}}\left(R_{h, \alpha} u-u, Z_{h, t}\right) d t,
$$

and, by Lemma 4.2 ,

$$
\begin{aligned}
\left|\sum_{n=1}^{N} \int_{J_{n}}\left(R_{h, \alpha} u-u, Z_{h, t}\right) d t\right| & \leq \max _{n \leq N}\left\|R_{h, \alpha} u-u\right\|_{J_{n}} \int_{0}^{t_{N}}\left\|Z_{h, t}\right\| d t \\
& \leq C L_{N} h^{2(r-\alpha)} \max _{n \leq N}\|u\|_{r, J_{n}}\|\varphi\|
\end{aligned}
$$

and similarly

$$
\begin{aligned}
\left|\sum_{n=1}^{N-1}\left(\rho^{n},\left[Z_{h}\right]_{n}\right)\right|+\left|\left(\rho^{N}, P_{h} \varphi\right)\right| & \leq \max _{n \leq N}\left\|\left(R_{h, \alpha} u-u\right)\left(t_{n}\right)\right\|\left(\sum_{n=1}^{N-1}\left\|\left[Z_{h}\right]_{n}\right\|+\left\|P_{h} \varphi\right\|\right) \\
& \leq C L_{N} h^{2(r-\alpha)} \max _{n \leq N}\|u\|_{r, J_{n}}\|\varphi\| .
\end{aligned}
$$

Finally, by the definition of $R_{h, \alpha}$,

$$
\begin{aligned}
\sum_{n=1}^{N} \int_{J_{n}} B_{\alpha}\left(\rho, Z_{h}\right) d t & =\sum_{n=1}^{N} \int_{J_{n}} B_{\alpha}\left(\tilde{u}-u, Z_{h}\right) d t \\
& =-\sum_{n=1}^{N} \int_{J_{n}}\left(A_{\alpha}(\tilde{u}-u), Z_{h}\right) d t=\sum_{n=1}^{N} K_{n}
\end{aligned}
$$

By the Assumption 3.1 and the definition of the interpolant $\tilde{u}$, we have

$$
\left|K_{n}\right| \leq k_{n}\|\tilde{u}-u\|_{r, J_{n}} \int_{J_{n}}\left\|Z_{h, t}\right\| d t
$$

Thus we have

$$
\sum_{n=1}^{N}\left|K_{n}\right| \leq \max _{n \leq N}\left(k_{n}\|\tilde{u}-u\|_{r, J_{n}}\right) \sum_{n=1}^{N} \int_{J_{n}}\left\|Z_{h, t}\right\| d t \leq C L_{N} \max _{n \leq N}\left(k_{n}^{3}\left\|u_{t t}\right\|_{r, J_{n}}\right)\|\varphi\| .
$$

Hence we get the following estimates

$$
\left|\left(\theta^{N}, \varphi\right)\right| \leq C L_{N} \max _{n \leq N}\left(k_{n}^{3}\left\|u_{t t}\right\|_{r, J_{n}}+h^{2(r-\alpha)}\right)\|\varphi\| .
$$

Together these estimates complete the proof of Theorem 4.3. 


\section{Numerical simulations}

In this section, we will consider two numerical examples.

Example 5.1. Consider the following linear space fractional partial differential equation, with $1 / 2<\alpha \leq 1$,

$$
\begin{aligned}
& \frac{\partial u(t, x)}{\partial t}-\frac{\partial^{2 \alpha} u(t, x)}{\partial|x|^{2 \alpha}}=f(t, x), \quad 0<t<T, 0<x<1, \\
& u(t, 0)=u(t, 1)=0, \quad 0<t<T, \\
& u(0, x)=u_{0}(x), \quad 0<x<1,
\end{aligned}
$$

where $u_{0}(x)=0$ and $f(t, x)=2 t x^{2}(1-x)^{2}-t^{2}\left(2-12 x+12 x^{2}\right)$. When $\alpha=1$, the exact solution is $u(t, x)=t^{2} x^{2}(1-x)^{2}$.

In the numerical simulation, we use the piecewise constant approximation in time and the linear finite element approximation in space. We consider the experimentally determined orders of convergence ("EOC") for the different $\alpha$ at $t_{n}=1$. We choose $k=0.001$ and the different space step size $h_{i}=1 / 2^{i}, i=1,2,3,4,5$. Let $e_{n}^{(i)}=\left\|u\left(t_{n}\right)-U^{n}\right\|_{L^{2}(0,1)}$ denote the $L^{2}$ norm at $t_{n}=1$ obtained by using the different space step sizes $h_{i}=1 / 2^{i}, i=1,2,3,4$. Since the exact solution is not available, we will calculate the reference solution ( or 'true' solution) $u\left(t_{n}\right)$ by using the very small time step size $k=0.0001$ and space step size $h=2^{-10}$. By Theorems 4.1, we have, with some $\alpha \leq r \leq 2 \alpha$ and $1 / 2<\alpha \leq 1$,

$$
e_{n}^{(i)} \leq C h_{i}^{r-\alpha}
$$

which implies that the convergence order $r-\alpha$ satisfies

$$
\log _{2}\left(e_{n}^{(i)} / e_{n}^{(i+1)}\right) \approx \log _{2}\left(h_{i} / h_{i+1}\right)^{r-\alpha}=r-\alpha .
$$

In Table 1, we observe that the experimentally determined orders of convergence ("EOC") are $2 \alpha$ which is much better than the theoretical convergence order in Theorem 4.1.

\begin{tabular}{|c|c|c|c|c|c|}
\hline$k$ & $\mathrm{~h}$ & $\mathrm{EOC}(\alpha=0.6)$ & $\mathrm{EOC}(\alpha=0.7)$ & $\mathrm{EOC}(\alpha=0.8)$ & $\mathrm{EOC}(\alpha=0.9)$ \\
\hline 0.001 & $1 / 2$ & & & & \\
\hline 0.001 & $1 / 4$ & 2.0132 & 2.4290 & 2.4232 & 2.3176 \\
\hline 0.001 & $1 / 8$ & 1.3547 & 1.6634 & 2.0163 & 2.1684 \\
\hline 0.001 & $1 / 16$ & 1.3493 & 1.3635 & 1.5023 & 1.5863 \\
\hline
\end{tabular}

Table 1: The experimentally determined orders of convergence ("EOC") for the different $\alpha$ at $t_{n}=1$ in Example 1

Example 5.2. Consider the following linear space fractional partial differential equation, with $1 / 2<\alpha \leq 1$,

$$
\begin{aligned}
& \frac{\partial u(t, x)}{\partial t}-\frac{\partial^{2 \alpha} u(t, x)}{\partial|x|^{2 \alpha}}=f(t, x), \quad 0<t<T, 0<x<1, \\
& u(t, 0)=u(t, 1)=0, \quad 0<t<T, \\
& u(0, x)=u_{0}(x), \quad 0<x<1,
\end{aligned}
$$

where $u_{0}(x)=x(1-x)$ and $f(t, x)=0$.

In Table 2, we observe that the experimentally determined orders of convergence ("EOC ") are also better than our theoretical convergence order $O\left(h^{r-\alpha}\right), \alpha \leq r \leq 2 \alpha$ in Theorem 4.1. We will investigate this issue in our future work. 


\begin{tabular}{|c|c|c|c|c|c|}
\hline$k$ & $\mathrm{~h}$ & $\mathrm{EOC}(\alpha=0.6)$ & $\mathrm{EOC}(\alpha=0.7)$ & $\operatorname{EOC}(\alpha=0.8)$ & $\mathrm{EOC}(\alpha=0.9)$ \\
\hline 0.001 & $1 / 2$ & & & & \\
\hline 0.001 & $1 / 4$ & 1.4233 & 1.5410 & 1.5249 & 1.4240 \\
\hline 0.001 & $1 / 8$ & 1.0621 & 1.1559 & 1.4353 & 1.6324 \\
\hline 0.001 & $1 / 16$ & 1.0171 & 1.1045 & 1.2011 & 1.5345 \\
\hline
\end{tabular}

Table 2: The experimentally determined orders of convergence ("EOC") for the different $\alpha$ at $t_{n}=1$ in Example 5.2

\section{References}

[1] B. Baeumer, M. Kovács, M. M. Meerschaert, Fractional reproduction-dispersal equations and heavy tail dispersal kernels, Bull. Math. Biol. 69(2007) 2281-2297.

[2] B. Baeumer, M. Kovács, M. M. Meerschaert, Numerical solutions for fractional reaction-diffusion equations, Comput. Math. Appl. 55(2008) 2212-2226.

[3] D. A. Benson, S. W. Wheatcraft, M. M. Meerschaert, Application of a fractional advection-dispersion equation, Water Resour. Res. 36(2000) 1403-1412.

[4] D. A. Benson, S. W. Wheatcraft, M. M. Meerschaert, The fractional order governing equations of Lévy motion, Water Resour. Res. 36(2000) 1413-1423.

[5] P. Biler, T. Funaki, W. A. Woyczynski, Fractal Burgers' equations, J. Differential Equations 148(1998) 9-46.

[6] W. Bu, Y. Tang, J. Yang, Galerkin finite element method for two-dimensional Riesz space fractional diffusion equations, Journal of Computational Physics 276(2014) 26-38.

[7] A. Bueno-Orovio, D. Kay, K. Burrage, Fourier spectral methods for fractional-in-space reaction-diffusion equations, BIT Numer. Math. (54)2014 937-954.

[8] K. Burrage, N. Hale, D. Kay, An efficient implicit FEM scheme for fractional-in-space reaction-diffusion equations, SIAM J. Sci. Comput. 34(2012) A2145-A2172.

[9] L. A. Caffarelli, A. Vasseur, Drift diffusion equations with fractional diffusion and the quasi-geostrophic equation, Ann. of Math. 171(2010) 1903-1930.

[10] A.S. Chaves, A fractional diffusion equation to describe Lévy flight, Physics Letters A, 239(1998) 13-16.

[11] W. Deng, Finite element method for the space and time fractional Fokker-Planck equation, SIAM J. Numer. Anal. 47(2008) 204-226.

[12] W. Deng, J. S. Hesthaven, Discontinuous Galerkin methods for fractional diffusion equations, ESAIM:M2AN, 47(2013) $1845-1864$.

[13] B. Duan, Z. Zheng, W. Cao, Finite element methods for a kind of two-dimensional space-fractional diffusion equation with its implementation, American Journal of Computational Mathematics 5(2015) 135-157.

[14] V. J. Ervin, J. P. Roop, Variational formulation for the stationary fractional advection dispersion equation, Numer. Methods Partial Differential Equations 22(2006) 558-576.

[15] V. J. Ervin, J. P. Roop, Variational solution of fractional advection dispersion equations on bounded domains in $\mathbb{R}^{d}$, Numer. Methods Partial Differential Equations 23(2007) 256-281.

[16] V. J. Ervin, N. Heuer, J. P. Roop, Numerical approximation of a time dependent nonlinear, space-fractional diffusion equation, SIAM J. Numer. Anal. 45(2007) 572-591.

[17] G.J. Fix, J. P. Roop, Least squares finite-element solution of a fractional order two-point boundary value problem, Comput. Math. Appl. 48(2004) 1017-1033.

[18] N. J. Ford, J. Xiao, Y. Yan, A finite element method for time fractional partial differential equations, Fract. Calc. Appl. Anal. 14(2011) 454-474.

[19] N. J. Ford, K. Pal, Y. Yan, An algorithm for the numerical solution of space-fractional partial differential equations, Computational Methods in Applied Mathematics 15(2015) 497-514.

[20] M. Ilic, F. Liu, I. Turner, V. Anh, Numerical approximation of a fractional-in-space diffusion equation I, Frac. Calc. Appl. Anal. 8(2005) 323-341.

[21] M. Ilic, F. Liu, I. Turner, V. Anh, Numerical approximation of a fractional-in-space diffusion equation II: with nonhomogeneous boundary conditions, Frac. Calc. Appl. Anal. 9(2006) 333-349.

[22] B. Jin, R. Lazarov, J. Pasciak, W. Rundell, Variational formulation of problems involving fractional order differential operators, Math. Comp. 84(2015) 2665-2700.

[23] B. Jin, R. Lazarov, J. Pasciak, Z. Zhou, Error analysis of finite element methods for space-fractional parabolic equations, SIAM J. Numer. Anal. 52(2014) 2272-2294.

[24] X. Leoncini, G. M. Zaslavsky, Jets, stickiness and anomalous transport, Phys. Rev. E, 65(046216), 2002.

[25] X. J. Li, C. J. Xu, A space-time spectral method for the time fractional diffusion equation, SIAM J. Numer. Anal. 47(2009) $2108-2131$.

[26] X. J. Li, C. J. Xu, Existence and uniqueness of the weak solution of the space-time fractional diffusion equation and a spectral method approximation, Commun. Comput. Phys. 8(2010) 1016-1051. 
[27] M. M. Meerschaert, C. Tadjeran, Finite difference approximations for fractional advection-dispersion flow equations, J. Comput. Appl. Math. 172(2004) 65-77.

[28] M. M. Meerschaert, H. Scheffler, C. Tadjeran, Finite difference methods for two-dimensional fractional dispersion equation, J. Comput. Phys., 211(2006) 249-261.

[29] R. Metzler, J. Klafter, The random walk's guide to anomalous diffusion: a fractional dynamics approach, Phys. Rep. $339(2000) 1-77$.

[30] K. Pal, Higher order numerical methods for solving fractional differential equations, PhD thesis, University of Chester, United Kingdom, 2015.

[31] I. Podlubny, Fractional Differential Equations, Academic Press, 1999.

[32] S. G. Samko, A. A. Kilbas, O.I. Marichev, Fractional Integrals and Derivatives: Theory and Applications (Translation from the Russian), Gordon and Breach, Amsterdam, 1993.

[33] J. P. Roop, Computational aspects of FEM approximation of fractional advection dispersion equations on bounded domains in $\mathbb{R}^{2}$, J. Comput. Appl. Math. 193(2006) 243-268.

[34] S. Shen, F. Liu, V. Anh, Numerical approximations and solution techniques for the space-time Riesz-Caputo fractional advection-diffusion equation, Numerical Algorithms 56(2011) 383-403.

[35] S. Shen, F. Liu, V. Anh, I. Turner, The fundamental solution and numerical solution of the Riesz fractional advectiondispersion equation, IMA J. Appl. Math. 73(2008) 850-872.

[36] E. Sousa, A second order explicit finite difference method for the fractional advection diffusion equation, Computers and Mathematics with Applications 64(2012) 3141-3152.

[37] C. Tadjeran, M. M. Meerschaert, H. Scheffler, A second-order accurate numerical approximation for the fractional diffusion equation, J. Comput. Phys. 213(2006) 205-213.

[38] C. Tadjeran, M. M. Meerschaert, A second-order accurate numerical method for the two-dimensional fractional diffusion equation, J. Comput. Phys. 220(2007) 813-823.

[39] V. Thomée, Galerkin Finite Element Methods for Parabolic Problems, Springer-Verlag, Berlin, 2006.

[40] Q. Yang, F. Liu, I. Turner, Numerical methods for fractional partial differential equations with Riesz space fractional derivatives, Appl. Math. Model. 34(2010) 200-218.

[41] H. Zhang, F. Liu, V. Anh, Galerkin finite element approximations of symmetric space-fractional partial differential equations, Appl. Math. Comput. 217(2010) 2534-2545.

[42] F. Zeng, F. Liu, C. Li, K. Burrage, I. Turner, V. Anh, Crank-Nicolson ADI spectral method for the two-dimensional Riesz space fractional nonlinear reaction-diffusion equation, SIAM J. Numer. Anal. 52(2014) 2599-2622. 